\title{
A Spiral Antenna Backed by a Conducting Plane Reflector
}

\author{
HISAMATSU NAKANO, MEMBER, IEEE, KAZUO NOGAMI, SATOSHI ARAI, HIROAKI MIMAKI, AND \\ JUNJI YAMAUCHI, MEMBER, IEEE
}

\begin{abstract}
An Archimedean planar spiral antenna is numerically analyzed in the presence of a conducting plane reflector. The analysis shows that the spiral antenna backed by the plane reflector has two distinct regions in the current distribution, which explain the radiation of a circularly polarized wave for the outer circumference $C$ ranging over about $1.3 \lambda<C<1.5 \lambda$ and $C>2.9 \lambda$, where $\lambda$ is a free-space wavelength. Further consideration is given to a truncated spiral antenna whose outer circumference is on the order of $1.4 \lambda$. The truncated spiral antenna maintains a decaying current distribution and radiates a circularly polarized wave over a 1:1.2 frequency bandwidth. It is also demonstrated that a power gain on the order of $8.5 \mathrm{~dB}$ is realized over the same frequency range.
\end{abstract}

\section{INTRODUCTION}

A SPIRAL ANTENNA [1]-[4] is usually required to radiate to one side only, or to have a unidirectional beam. This is accomplished by appropriately backing the spiral antenna on one side with an electromagnetic wave absorber, a cavity, or a conducting plane reflector. For use over an extremely wide band, for instance, of 1:10, the spiral antenna is usually backed by the absorber, where only half of the power is radiated [5]. On the other hand, for use over a relatively narrow bandwidth, the spiral antenna may be backed by a cavity [6] or a conducting plane reflector. In the former spiral backed by the cavity it is experimentally found that the axial ratio of less than $3 \mathrm{~dB}$ is maintained over an octave frequency range. In the latter spiral backed by the plane reflector, little open literature is available, and the plane reflector has been used on the basis of experience only.

The motivation of this research arises from a desire to reveal theoretically the radiation characteristics of a spiral antenna with a conducting plane reflector. The antenna characteristics which are evaluated from the numerically obtained current distributions, not the assumed current distributions [1]-[3], are presented with some experimental data.

\section{Antenna Geometry}

Fig. 1 illustrates the antenna configuration and coordinate system. The antenna arm is characterized by Archimedean spiral function $r=a \phi_{s}$ ( $a$ :spiral constant, $\phi_{s}$ :winding angle).

Manuscript received September 25, 1985; revised December 16, 1985.

H. Nakano, K. Nogami, S. Arai and H. Mimaki are with the Department of Electrical Engineering, College of Engineering, Hosei University, 3-7-2 Kajino-cho, Koganei-City, Tokyo 184, Japan.

J. Yamauchi is with the Department of Electrical Engineering, Tokyo Metropolitan Technical College, 1-10-40 Higashi-Ooi, Shinagawa-ku, Tokyo 140, Japan.

IEEE Log Number 8608087.
In order to operate the spiral antenna as a quasiself-complementary antenna [7], we choose the geometrical parameters in such a way that the ratio of the equivalent strip width $4 \rho$ $(\rho:$ wire radius) to the spacing width between the neighboring wires is unity. The parameters are as follows: spiral constant $a$ $=0.764 \mathrm{~mm}$; wire radius $\rho=0.3 \mathrm{~mm}$; feed wire length $e_{s t}=$ $2 \mathrm{~mm}$; range of winding angle $\phi_{s} 2.6 \mathrm{rad} \leq \phi_{s} \leq 36.5 \mathrm{rad}$.

The spiral antenna is backed by a perfectly conducting plane reflector of infinite extent, and handled by the method of image in the present analysis.

The current distribution along the spiral arm is determined by using an integral equation [8] with the moment method [9]. The input impedance, radiation pattern, axial ratio and power gain are calculated on the basis of the obtained current distribution.

\section{Antenna Characteristics as a Function of the Outer CirCumference}

In this section, we investigate the antenna characteristics as a function of the outer circumference $C$ of the spiral antenna, with the operating frequency fixed at $6 \mathrm{GHz}$. The analysis is made over an outer circumference range between $1.1 \lambda$ and $3.5 \lambda(\lambda=5 \mathrm{~cm})$. The spiral antenna is placed at a distance of $d=1.25 \mathrm{~cm}(=\lambda / 4)$ from the plane reflector, and excited by a delta-gap voltage source of $1 \mathrm{~V}$.

\section{A. Current Distribution}

Fig. 2 shows the theoretical current distribution $\left(I=I_{r}+\right.$ $\left.j I_{i}\right)$ and its phase progression. In Fig. 2(a) the antenna arm length is $50.8 \mathrm{~cm}$, making the outer circumference of the spiral antenna $3.5 \lambda$ long. As additional information, the current amplitude $|I|$ of the corresponding isolated spiral antenna of the same parameters without the plane reflector is also presented at the bottom. The regions with average circumferences of one wavelength and three wavelengths, where the first and third radiation bands [2] appear, are marked by arrows.

There exists a noticeable difference between the two current distributions at the top and the bottom in Fig. 2(a). Owing to the mutual coupling between the spiral antenna and its image antenna, the current distribution of the spiral antenna backed by the plane reflector is divided into two distinct regions. One is a decaying region from the feed point to the point of the first minimum indicated by a double arrow. The other is a residual current region appearing after the establishment of the first minimum point. In both regions the current travels with a 

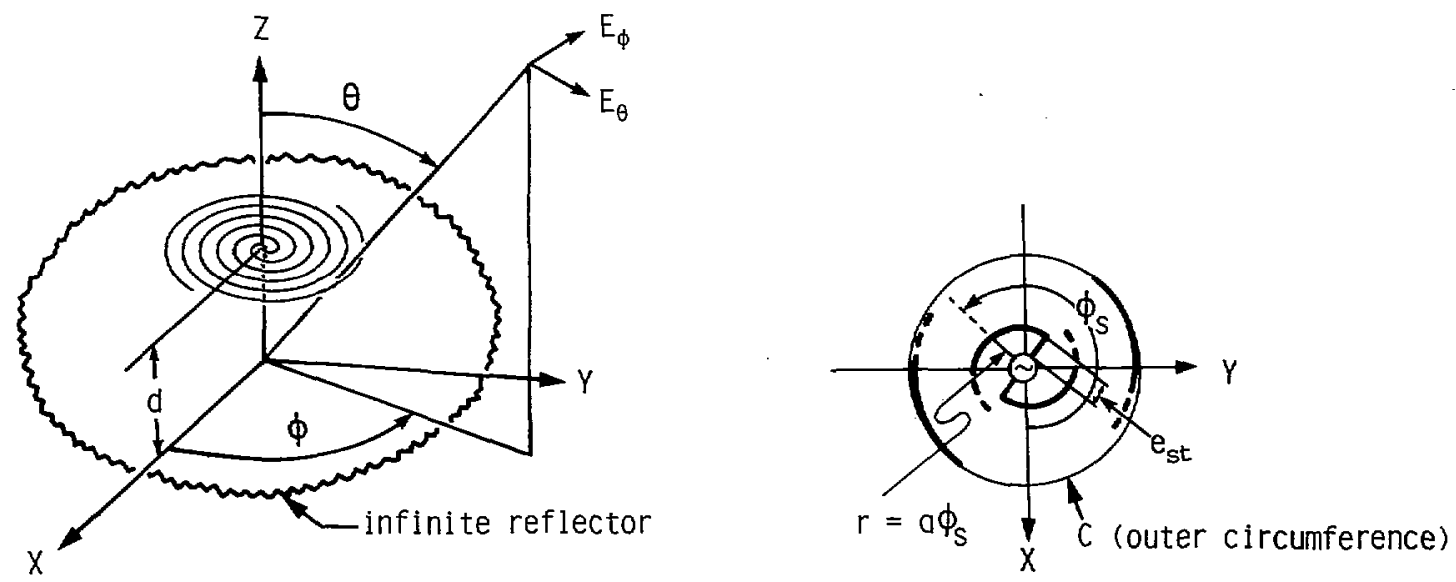

Fig. 1. Antenna configuration and coordinate system.

phase velocity nearly equal to the velocity of light in free space. Further calculations show that the two regions clearly exist provided that the circumference of the spiral antenna is more than $2.9 \lambda$. This property resulting from the conducting plane reflector, or the coupling between the real antenna and its image, is observed in other antennas such as a monofilar conical-helix antenna [10].

Figs. 2(b) and 2(c) show the current distributions with arm lengths of $16.4 \mathrm{~cm}$ and $8.4 \mathrm{~cm}$, corresponding to the outer circumferences of about $C=2.0 \lambda$ and $C=1.4 \lambda$, respectively. In the case of $C=2.0 \lambda$ the current distribution indicates a standing wave form, losing the linearity in the phase progression. It should be noted, however, that the spiral antenna with $C=1.4 \lambda$ has only a smoothly decaying current, which travels with a velocity nearly equal to the velocity of light. In addition, it is noted that the decaying in the current of the spiral antenna backed by the plane reflector is smoother than that of the corresponding isolated spiral antenna without the plane reflector. This contributes to better radiation of a circularly polarized wave, as will be mentioned in Section IIIB.

As long as a decaying current distribution is established, the input impedance indicates a nearly pure resistance of about $200 \Omega$. This resistance value is close to the impedance of $60 \pi$ $\Omega$ of a self-complementary antenna. It is also worth mentioning that the input impedance is almost the same as that observed in the isolated spiral antenna without the plane reflector.

\section{B. Axial Ratio}

Fig. 3 shows the behavior of the axial ratio in the $+Z$ axis direction as a function of the outer circumference $C$. For comparison, the axial ratio of the isolated spiral antenna without the plane reflector is also plotted in this figure.

In contrast with a monotonic change observed in the isolated spiral antenna, the axial ratio of the spiral antenna backed by the plane reflector varies complicatedly. It is found that the spiral radiates a circularly polarized wave with an axial ratio of less than $3 \mathrm{~dB}$ for the outer circumference of more than $2.9 \lambda$. It is also found that although the radiated wave becomes elliptically polarized with a decrease in the outer circumference $C$, it again becomes circularly polarized over an outer circumference range from $1.3 \lambda$ to $1.5 \lambda$. An axial ratio at $C$ $=1.4 \lambda$ is calculated to be $2.0 \mathrm{~dB}$, which is better than the axial ratio of the corresponding isolated spiral antenna without the plane reflector.

Although it is difficult to explain the mechanism of the axial ratio qualitatively because of the complicated mutual coupling between the spiral antenna and its image, the change in the axial ratio is closely related to the behavior of the current distribution. Since a decaying current distribution is realized provided that the outer circumference $C$ is in ranges of about $1.3 \lambda<C<1.5 \lambda$ and $C>2.9 \lambda$ as described in Section III-A, a circularly polarized wave is generated in these two ranges of the outer circumference. For other circumferences a retrogressive current, causing the total current distribution to be a standing wave form together with a progressive current, deteriorates the axial ratio, because the rotational sense of a circularly polarized wave radiated from the retrogressive current traveling toward the input of the spiral is opposite to that of a circularly polarized wave radiated from the progressive current traveling toward the arm end.

\section{Radiation Pattern and Power Gain}

Figs. 4(a), 4(b), and 4(c) illustrate typical radiation patterns of the spiral antennas whose outer circumferences are $3.5 \lambda$, $2.0 \lambda$, and $1.4 \lambda$, respectively, together with those of the isolated spiral antennas without the plane reflector. Each of the radiation patterns of the isolated spiral antennas is symmetrical with respect to the spiral antenna plane, and hence only half of the radiation pattern is illustrated.

In the spiral antenna backed by the plane reflector the $E_{\phi}$ component of the radiation field vanishes in the direction of $\theta$ $= \pm 90^{\circ}$, due to the fact that the spiral antenna and its image have currents of equal magnitude but of opposite phase. The vanishing of $E_{\phi}$ at $\theta= \pm 90^{\circ}$, resulting in a narrower beam, and the unidirectional radiation lead to an increase in the power gain.

For the outer circumference of $C=3.5 \lambda$ the gain of the spiral antenna increases to $8.7 \mathrm{~dB}$ from a value of $6.0 \mathrm{~dB}$ for the corresponding isolated spiral antenna without the plane reflector. When the outer circumference $C$ is $1.4 \lambda$, the spiral antenna over a plane has a gain of $8.4 \mathrm{~dB}$, while the 


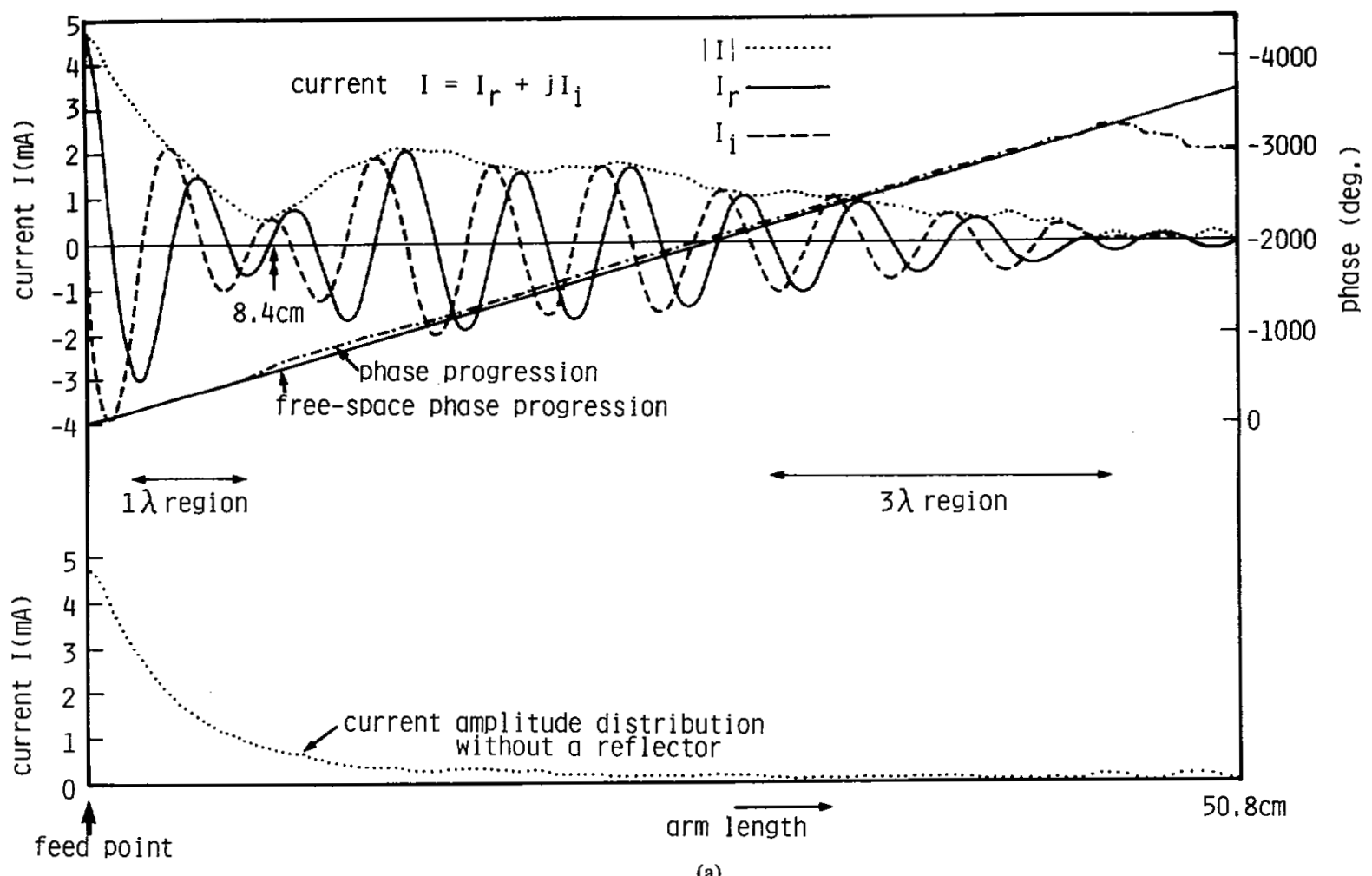

(a)

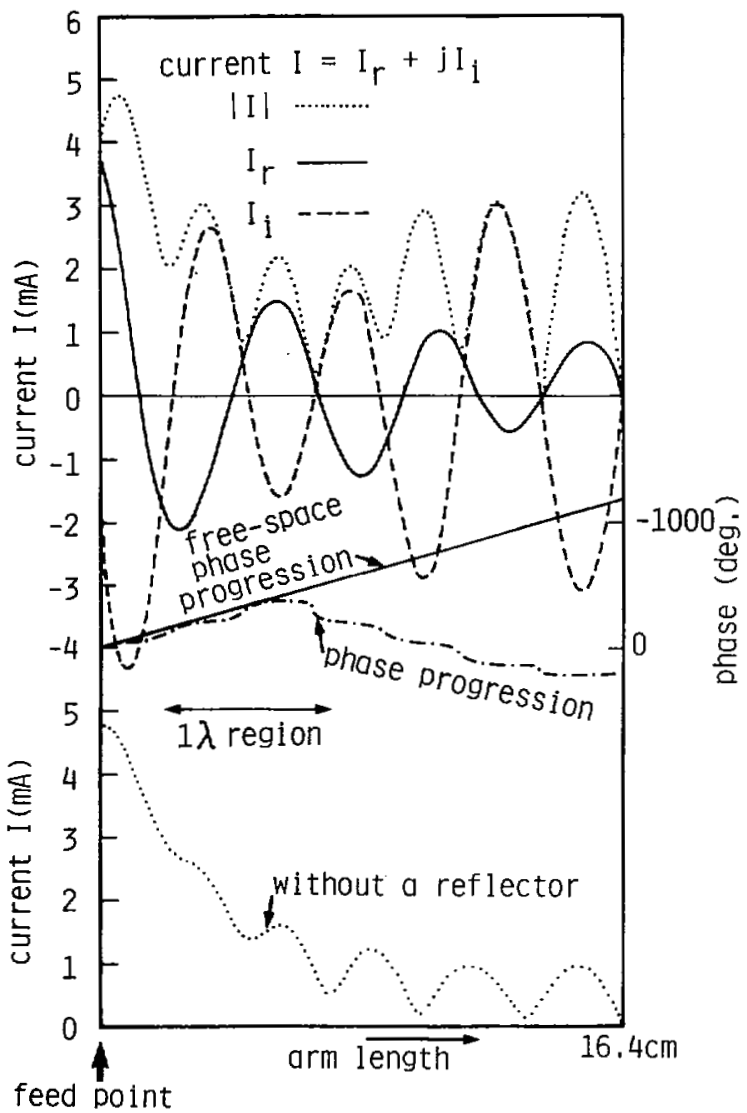

(b)

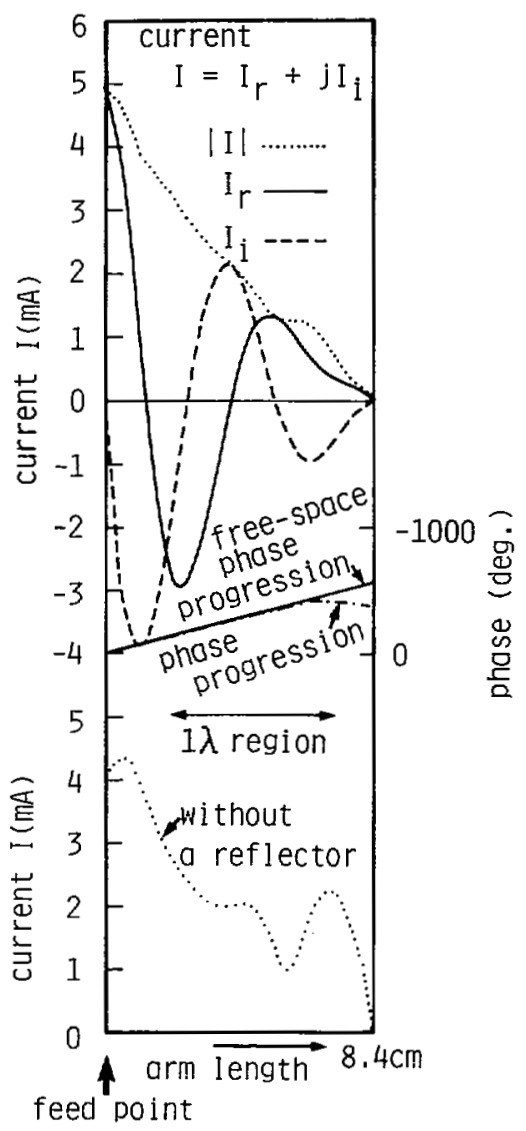

(c)

Fig. 2. Theoretical current distributions for (a) $C=3.5 \lambda$. (b) $C=2.0 \lambda$. (c) $C=1.4 \lambda$. 


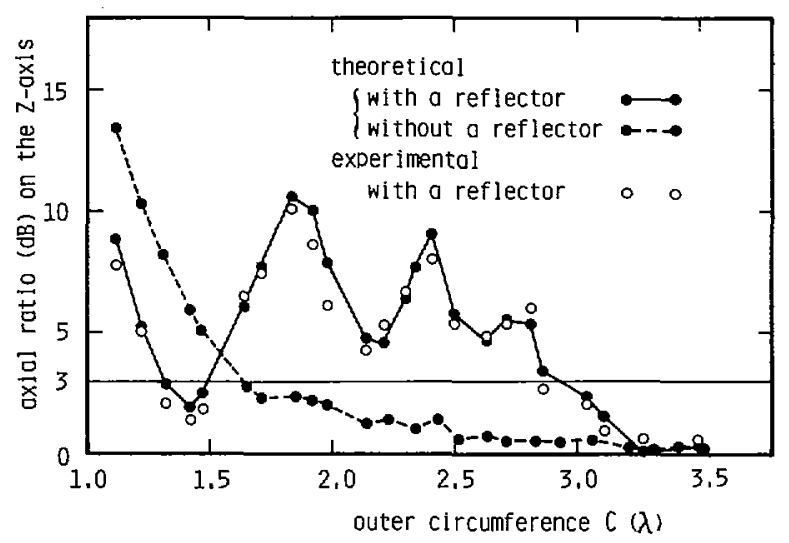

Fig. 3. Axial ratio as a function of the outer circumference.

$$
\begin{array}{ll}
\text { experimental } & \text { theoretical } \\
\left\{\begin{array}{lll}
E_{\theta} \times x & \left\{\begin{array}{l}
E_{\theta}- \\
E_{\phi}---
\end{array}\right.
\end{array}\right.
\end{array}
$$

with a reflector

without a reflector
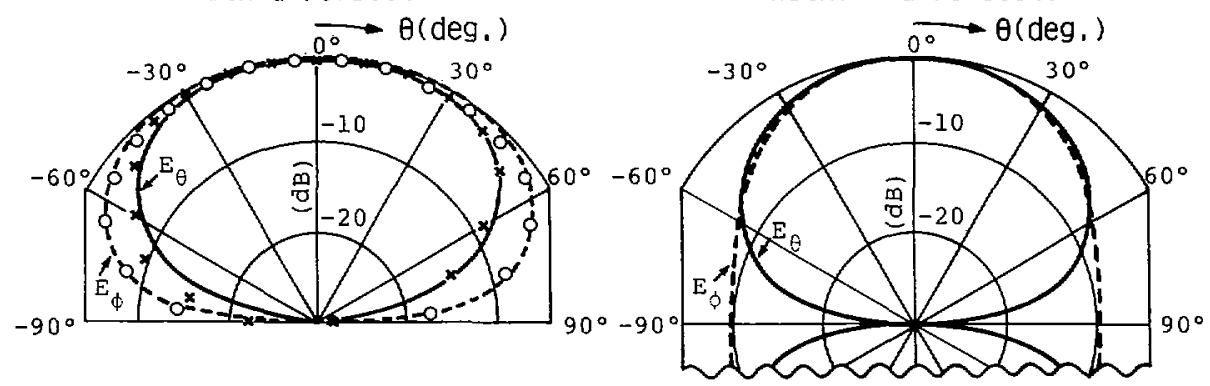

(a)

with a reflector
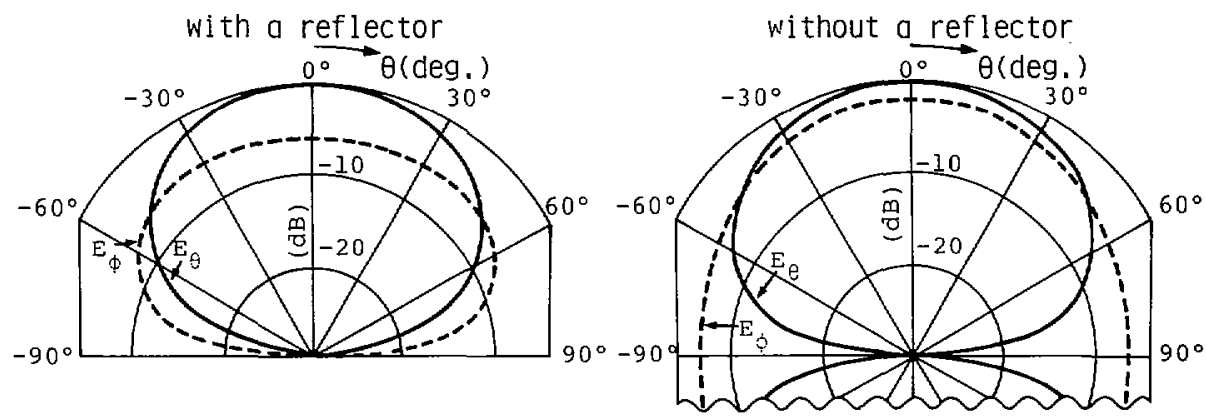

(b)

with a reflector

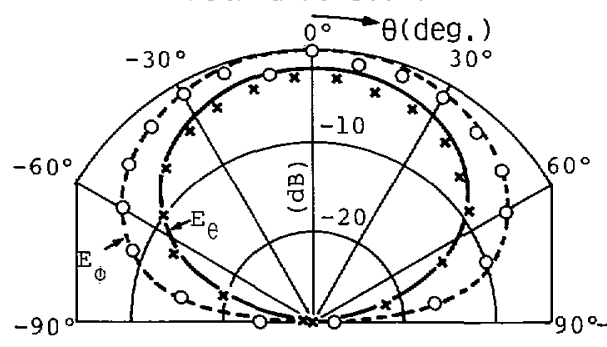

without a reflector

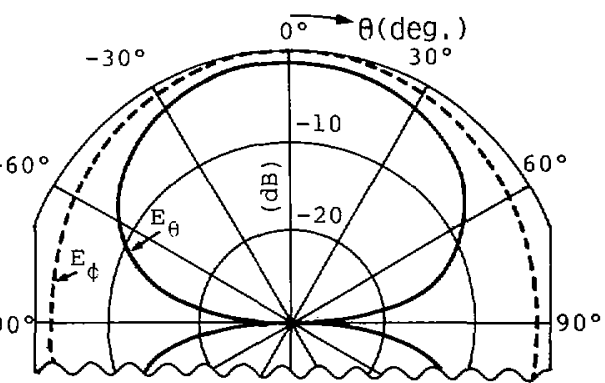

(c)

Fig. 4. Radiation patterns in $\phi=0^{\circ}$ plane. (a) Outer circumference $C=3.5 \lambda$. (b) Outer circumference $C=2.0 \lambda$. (c) Outer circumference $C=1.4 \lambda$ 


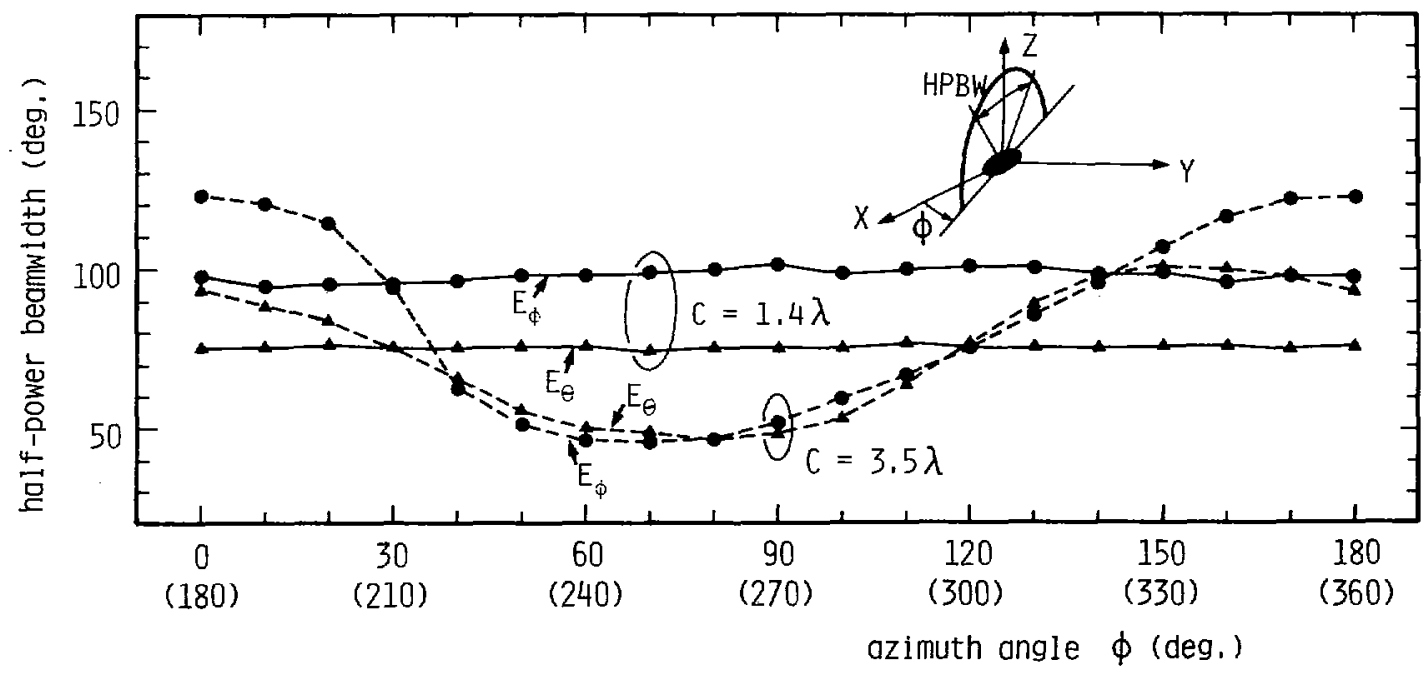

Fig. 5. Half-power beamwidth as a function of the azimuth angle.

corresponding isolated spiral antenna has a gain of $3.9 \mathrm{~dB}$. It is noted that the gain of the spiral antenna backed by a plane reflector remains nearly constant for outer circumference ranges of about $1.3 \lambda<C<1.5 \lambda$ and $C>2.9 \lambda$.

Examination of the radiation pattern for $C=3.5 \lambda$ reveals that the half-power beamwidth (HPBW) remarkably changes as a function of the azimuth angle $\phi$, as shown in Fig. 5. For instance, the maximum HPBW of the $E_{\phi}$ component is $124^{\circ}$ at $\phi=0^{\circ}$, while the minimum HPBW is $46^{\circ}$ at $\phi=70^{\circ}$. On the other hand, the HPBW for $C=1.4 \lambda$ shows little variation regardless of the change in the azimuth angle. In the following section the latter spiral antenna of $C=1.4 \lambda$ is further investigated for practical applications.

\section{Frequency Characteristics of a Truncated Spiral antenna Backed by a Plane Reflector}

It has been found that a truncated spiral antenna consisting of only the decaying current region is attractive from the point of view of the pattern symmetry with respect to the antenna axis. In this section, some consideration is given to the frequency characteristics of a truncated spiral antenna in which the outer circumference $C$ is $7 \mathrm{~cm}$, corresponding to 1.4 $\lambda$ at $6 \mathrm{GHz}$. The distance $d$ between the spiral and the plane reflector is again fixed to be $1.25 \mathrm{~cm}$.

Calculations are made over a range of frequencies of 5.0 $\mathrm{GHz}$ to $6.5 \mathrm{GHz}$, where the distance $d$ is changed from $0.21 \lambda$ to $0.27 \lambda$ in terms of the operating wavelength. There is essentially no difference in the current distributions, which decay smoothly from the feed point toward the arm end, as in Fig. 2(c). It is also found that the current at the input terminal remains relatively constant, and hence a wide-band characteristic of the input impedance is obtained as shown in Fig. 6. Over the frequency range of $5.5 \mathrm{GHz}$ to $6.5 \mathrm{GHz}$, its resistance is on the order of $200 \Omega$ with a variation of only about $3 \Omega$, while the reactance is on the order of $16 \Omega$ with a similar variation of 3 $\Omega$.

Fig. 7 shows the frequency characteristics of the power gain. No significant change in the gain is observed, since the radiation pattern remains nearly unchanged regardless of the

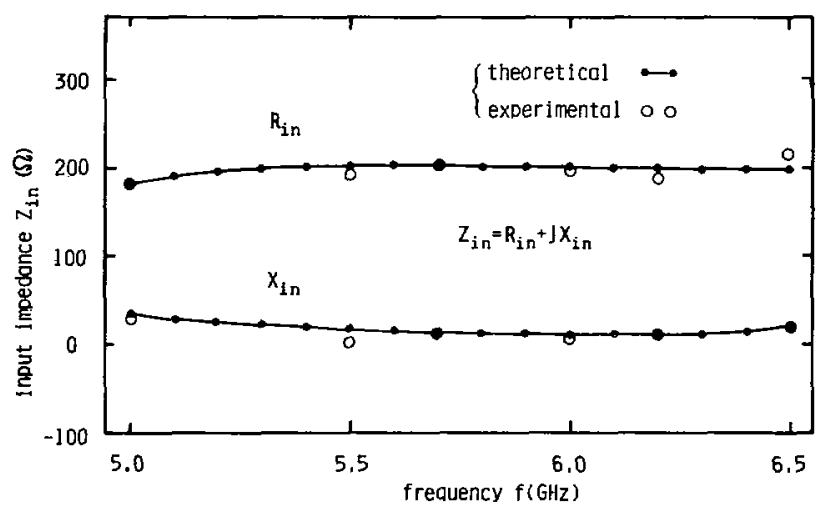

Fig. 6. Input impedance as a function of frequency.

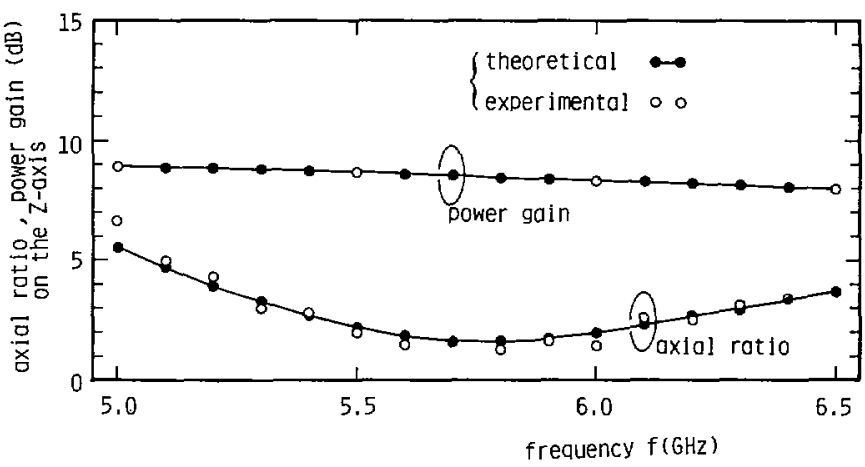

Fig. 7. Power gain and axial ratio as a function of frequency.

change in frequency. An increase of more than $4.0 \mathrm{~dB}$ from the gain of the corresponding isolated spiral antenna without the plane reflector is obtained over a range of frequencies from $5.0 \mathrm{GHz}$ to $6.5 \mathrm{GHz}$.

Fig. 7 also shows the axial ratio characteristics. An axial ratio of less than $3 \mathrm{~dB}$ is obtained over a range of frequencies from $5.35 \mathrm{GHz}$ to $6.30 \mathrm{GHz}$ (1:1.2) owing to the smoothness of the current distribution.

\section{Conclusion}

A spiral antenna backed by an infinite plane reflector radiates a circularly polarized wave with an axial ratio of less 
than $3 \mathrm{~dB}$, when the outer circumference $C$ is in ranges of about $1.3 \lambda<C<1.5 \lambda$ and $C>2.9 \lambda$. It is noted that the half-power beamwidth for $C>2.9 \lambda$ is dependent on the azimuth angle, whereas the HPBW for $1.3 \lambda<C<1.5 \lambda$ is almost independent of the azimuth angle.

In a truncated spiral antenna whose outer circumference is on the order of $1.4 \lambda$, it is revealed that a circularly polarized wave is radiated over a 1:1.2 range in frequency, where the input impedance indicates a nearly pure resistance of about $200 \Omega$. The power gain of the spiral antenna placed at a distance of $\lambda / 4$ from the plane reflector is found to be increased by about $4 \mathrm{~dB}$ from that of the corresponding isolated spiral antenna without the plane reflector.

\section{REFERENCES}

[1] W. L. Curtis, "Spiral antennas," IRE Trans. Antennas Propagat., vol. AP-8, pp. 298-306, May 1960.

[2] J. A. Kaiser, "The Archimedean two-wire spiral antenna," IRE Trans. Antennas Propagat., vol. AP-8, pp. 312-323, May 1960.

[3] H. D. Cubley and H. S. Hayre, "Radiation fields of spiral antennas employing multimode slow wave techniques," IEEE Trans. Antennas Propagat., vol. AP-19, pp. 126-128, Jan. 1971.

[4] H. Nakano and J. Yamauchi, "Characteristics of modified spiral and helical antennas," Inst. Elec. Eng. Proc., pt. H, vol. 129, pp. 232237, Oct. 1982.

[5] R. C. Johnson and H. Jasik, Eds., Antenna Engineering Handbook. New York: McGraw-Hill, 1984.

[6] R. C. Hansen, Ed., Microwave Scanning Antennas, vol. 2, Array Theory and Practice. New York: Academic, 1966, pp. 116-127.

[7] V. H. Rumsey, Frequency Independent Antennas. New York: Academic, 1966.

[8] H. Nakano, "The integral equations for a system composed of many arbitrarily bent wires," Trans. IECE Japan., vol. E65, pp. 303-309, June 1982.

[9] R. F. Harrington, Field Computation by Moment Methods. New York: Macmillan, 1968.

[10] H. Nakano, T. Mikawa, and J. Yamauchi, "Monofilar conical-helix antenna with low pitch angle," Inst. Elec. Eng. Proc., pt. H, vol. 131, pp. 379-382, Dec. 1984.

Hisamatsu Nakano (M'75), for a photograph and biography please see page 840 of the August 1984 issue of this TRANSACTIONS.

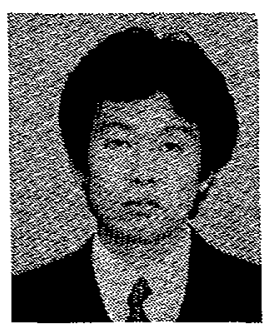

Kazuo Nogami was born in Tokyo, Japan, on April 16, 1960. He received the B.E. and M.E. degrees in electrical engineering from Hosei University, in 1983 and 1985, respectively.

He joined the Toshiba Co. Ltd., Kawasaki, in 1985.

Mr. Nogami is a member of the Institute of Electronics and Communication Engineers of Japan.

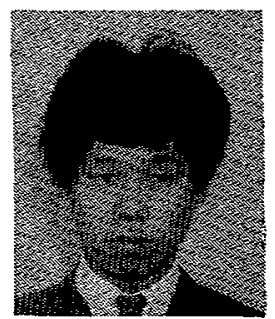

Satoshi Arai was born in Tokyo, Japan, on June 21,1962 . He received the B.E. degree in electrical engineering from Hosei University in 1985 . He is now engaged in studies of the electrical engineering for the M.E. degree at Hosei University.

Mr. Arai is a member of the Institute of Electronics and Communication Engineers of Japan.

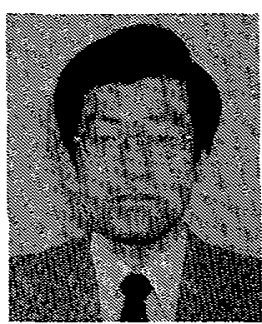

Hiroaki Mimaki was born in Yamaguchi, Japan, on December 7, 1952. He received the B.E. degree in electronic engineering from Tokyo Denki University, Tokyo, in 1976 and M.E. degree in electrical engineering from Hosei University, Tokyo, in 1981.

$\mathrm{He}$ is currently an assistant at Hosei University. His research interests are thin wire antennas.

Mr. Mimaki is a member of the Institute of Electronics and Communication Engineers of Japan.

Junji Yamauchi (M'85), for a photograph and biography please see page 840 of the August 1984 issue of this TRANSACTIONS. 